Brief

Report

\title{
Agent-based model of laser hair removal: A treatment optimization and patient education tool
}

\author{
Bell Raj Eapen
}

Kaya Skin Clinic, Dubai, UAE

Address for correspondence: Dr. Bell Raj Eapen, Kaya Skin Clinic - SBC, PB No - 50394, Dubai, U.A.E. E-mail:

webmaster@gulfdoctor.net

DOI: $10.4103 / 0378-6323.53135$

\begin{abstract}
Background: Tracking of various parameters associated with laser hair removal is tedious and time consuming. The currently available mathematical models are not simple enough for physicians to be used as a treatment optimization and patient education tool. Aim: The aim of the study was to develop a mathematical model for laser hair removal using agent-based modeling and to make a user-friendly simulation environment. Methods: The model was created using NetLogo. The hairs were modeled as agents oscillating between anagen and telogen. The variables were assigned based on published data whenever possible and the various paths the agent could take were coded as conditional statements. The improvement was assessed using an arbitrary index which takes into account the mean diameter and pigmentation along with the number and length of hairs visible above the surface. Few of the commonly encountered scenarios were simulated using the model. Results: The model is made freely available online (http://www.gulfdoctor.net $/$ model $/ \mathrm{lhr}$.htm). Limited number of simulations performed indicated that an eight-week gap between laser sessions may be more effective than a four-week gap. Conclusions: The simulation provides a reliable tool for treatment optimization and patient education as obtaining relevant clinical data is slow and labor-intensive. Its visual interface and online availability makes it useful for everyday use.
\end{abstract}

Key words: Laser hair removal, Agent based model

\section{INTRODUCTION}

Laser hair removal (LHR) has emerged as a popular cosmetic dermatology procedure. Several types of lasers are available for hair removal along with non-laser intense pulse light (IPL) epilators. ${ }^{[1]}$ However, optimizing these devices according to the skin and hair type of the patient, to maximize results and minimize adverse effects is a difficult task.

Laser hair removal is a slow process which takes at least six to eight months. The apparent cosmetic improvement may be related to reduction in number of hairs or a decrease in diameter or pigmentation. ${ }^{[2]}$ Tracking of these parameters over such long periods is tedious and impractical. Hence a lot of confusion still exists regarding the optimum settings and the type of laser most beneficial for a given hair and skin type. ${ }^{[3]}$

Since the 'selective photothermolysis' induced by laser only destroys a small fraction of hairs completely, lasers can achieve only permanent hair reduction and a certain percentage of hair will always grow back. ${ }^{[4]}$ Conveying this message as well as a frank discussion of risk-benefit ratio with the patient is demanding but imperative.

Mathematical models have been developed to simulate LHR for research purposes. ${ }^{[5,6]}$ Some models have even succeeded in deducing epilation strategies based on simulation. However, none of these models is simple enough to be used by a laser practitioner as a patient education tool. Hence we identified the need for an LHR simulation model which is sensitive enough to reliably predict its long-term effects and simple enough to be used as a patient education tool.

We chose 'Agent-based modeling' $(\mathrm{ABM})^{[7]}$ using NetLogo ${ }^{[8,9]}$ as a platform for LHR modeling as it can effectively hide complexities of the model behind an intuitive visual interface. Agent-based modeling simulates the behavior of discrete units separately

How to cite this article: Eapen BR. Agent-based model of laser hair removal: A treatment optimization and patient education tool. Indian J Dermatol Venereol Leprol 2009;75:383-7.

Received: November, 2008. Accepted: January, 2009. Source of Support: Nil. Conflict of Interest: None declared. 
and evaluates the impact on the system as a whole. ${ }^{[7]}$ This is ideal for modeling LHR as each hair behaves as distinct, non-deterministic units cycling through anagen, telogen and catagen. The probabilities for normal behaviors like phase switching and responses towards external perturbations like laser administration are entered into the model as conditional statements (if-then statements).

We have used probability data from published experimental studies whenever possible. Certain parameters are deduced from related data or assumed from clinical experience. However these parameters can be easily altered in the model.

NetLogo visual interface consists of agents, traditionally called 'turtles' interacting with the background called 'patches'. An 'observer' sets the turtle and patch parameters using 'sliders' or 'choosers' and monitors the output which can be textual (monitors) or graphs (plots). NetLogo environment, being Java based has the added advantage of running directly in a web browser. The model including the visual interface is available from http://www.gulfdoctor.net/model/lhr.htm.

The scoring of hirsutism like the Ferriman-Gallway score $^{[10]}$ is not sensitive enough to capture subtle changes in a mathematical model. Besides, patients without overt hirsutism can also undergo LHR for cosmetic reasons. Hence an arbitrary index was used for assessment of improvement as explained in methods.

The visual interface consists of a $1 \mathrm{~cm}^{2}$ skin surface. The input parameters for hair include the hair density and percentages of thick and pigmented hair all of which can be altered using slider controls. All laser parameters and the skin type can also be altered. Wavelength can be chosen from that of three popular lasers used for hair removal (NdYAG, Diode and Alexandrite). The output consists of mean hair length, pigmentation, thickness and the percentage of anagen and telogen hairs. The improvement index, as explained in methods is displayed as a plot and the adverse effects during each session are shown. Pressing the setup key initializes the system and it is the first step before running the simulation. Pressing the 'Go' button will start the simulation. If the 'give-laser' key is turned on the specified number of laser sessions will be administered. There is a separate key to administer a single session of laser during any stage of the simulation. Any of the parameters can be altered either before or during simulation. The speed of simulation can be controlled by the slider in the toolbar and the number of days is displayed on top of the visual area.

\section{METHODS}

The hairs are modeled as discrete 'agents' oscillating between anagen and telogen phase. Since the percentage of catagen hair is less than the other two, it is not included in the model. The hair parameters like density and the percentage of thick and dark hairs can be set using the sliders. The visual area represents one square centimeter of skin surface. The hairs are color coded according to the growth phase and laser damage (grey $=$ anagen; yellow $=$ telogen; red $=$ damaged by laser). The background (patch) represents the skin which is brown for skin types 1-3 and black for skin types 4-6. All the important laser parameters, such as wavelength, fluence, pulse width, pulse duration, spot size and gap between two sessions can be adjusted with sliders.

The initial random phase assignment as anagen and telogen is based on the normal A : T ratio of 70:30. ${ }^{[1]}$ The anagen hair grows at a rate of $0.24 \mathrm{~mm}$ per day (SD 0.07). The randomly assigned maximum age of anagen hairs have a mean of 177 days (SD 32) and telogen hairs have a mean of 91 days (SD 16). ${ }^{[6]}$ The hair undergoes phase switching once the assigned maximum age is reached.

Laser is given at preset intervals once the 'give-laser' switch is turned on. The probabilities of hair and skin injury are calculated according to formula (1) and (2), respectively.

Thickness x Pigmentation $\mathrm{x}$
Fluence $\mathrm{x}$ Spot size $\mathrm{x}$
$\frac{\text { Pulse duration }}{\text { Wave length } \mathrm{x} \text { Pulse width }}=$ A x Hair damage ... (1)

Skin type $\mathrm{x}$ Fluence $\mathrm{x}$

Spot size $\mathrm{x}$ Pulse duration $=$ B $\mathrm{x}$ Skin damage ..(2)

Wave length $x$ Pulse width

This is based on the assumption that hair and skin damage is directly proportional to pigmentation, ${ }^{[12]}$ laser fluence, pulse duration ${ }^{[13]}$ and spot size ${ }^{[14]}$ and inversely proportional to wavelength, ${ }^{[15]}$ pulse width. ${ }^{[16]}$ The hair damage is also proportional to the thickness of hair. ${ }^{[17]}$ The thickness and pigmentation of the hair were graded as 1 and 2 . The proportionality constants $\mathrm{A}$ and $\mathrm{B}$ were roughly approximated to $4 \times 10^{-4}$ and $3 \times 10^{-4}$, respectively based on available clinical data. ${ }^{[18]}$ 
However these values are rough approximations and can be easily altered in the model.

An injured hair can be either completely destroyed or partially injured. Partial injury can result in reduction in pigmentation or a reduction in thickness. ${ }^{[5]}$ In case of anagen hairs the probability of an anagen to telogen conversion is also included.

Each cycle represents one day. A normal hair switches phase at the appropriate time, check the effect of laser if administered, recover from any pre-existing injury and grow during each cycle. A schematic representation of the model is shown in [Figure 1].
The skin injury is further characterized according to the published data as erythema, pain, folliculitis, pigmentary changes, crusting and superficial scarring. Deep irreversible scarring is very rare and was not included in the model. ${ }^{[19]}$

The improvement was calculated by subtracting final hirsutism index from initial hirsutism index and the percentage of improvement was calculated and displayed as a plot. The arbitrary hirsutism index was calculated as per formula (3).

$(5 \times$ Surface hair $)+(3 \times$ Mean thickness $)+(2 \times$ Mean pigmentation) + Mean length

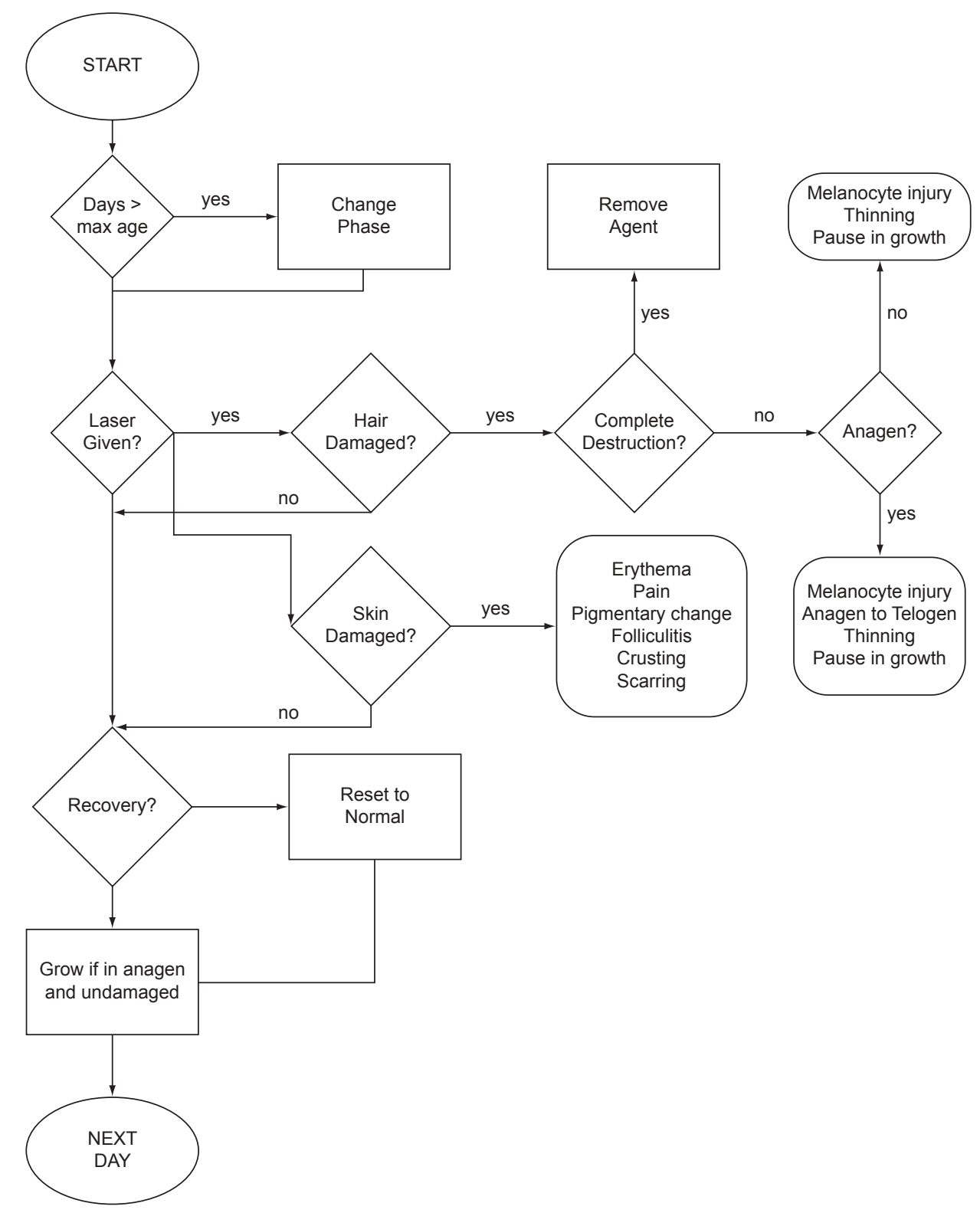

Figure 1: Flow chart of the probable paths an agent can take during simulation 
Hair and skin injury sustained have a preset time period following which the injury heals and the hair returns back to normal state. This can lead to gradual increase in the index once the laser sessions are completely stopped.

\section{RESULTS}

The simulation was performed with pre-determined settings, ten iterations for each type of laser for duration of two years. The fluence of $50 \mathrm{~J} / \mathrm{cm}^{2}$ and a pulse width of $20 \mathrm{~ms}$ were used for all simulations. The results are summarized in Table 1. As the data is an output of simulation, a detailed statistical analysis was not performed. The plot of improvement

\begin{tabular}{lccccc}
\hline \multicolumn{5}{c}{ Table 1: Results of simulation } \\
\hline $\begin{array}{l}\text { Wave } \\
\text { length }\end{array}$ & $\begin{array}{c}\text { Gap } \\
\text { between } \\
\text { sessions }\end{array}$ & $\begin{array}{l}\text { Skin } \\
\text { type }\end{array}$ & & $\begin{array}{c}\text { Improvement } \\
\text { Mean no. } \\
\text { of transient } \\
\text { adv effects* }\end{array}$ & $\begin{array}{c}\text { Mean no. of } \\
\text { persistent } \\
\text { adv effects }\end{array}$ \\
\hline 1064 & 4 & 4 & 37.5 & 4.2 & 0.5 \\
810 & 4 & 4 & 40.68 & 5 & 0.6 \\
755 & 4 & 4 & 41.69 & 4.9 & 0.2 \\
1064 & 8 & 4 & 37.88 & 2.8 & 0.1 \\
810 & 8 & 4 & 42.96 & 4.3 & 0.4 \\
755 & 8 & 4 & 44.75 & 5.1 & 0.5 \\
1064 & 4 & 5 & 36.3 & 3.8 & 0.2 \\
810 & 4 & 5 & 41.24 & 4.9 & 0.3 \\
755 & 4 & 5 & 42.26 & 5.5 & 1.1 \\
\hline $\begin{array}{l}{ }^{*} \text { Pain, erythema and folliculitis; **Pigmentary changes, crusting and superficial } \\
\text { scarring }\end{array}$ & \multicolumn{5}{l}{}
\end{tabular}

percentage against days during one of the simulations is shown in [Figure 2].

\section{DISCUSSION}

The results of the simulation confirms with the available clinical data. ${ }^{[18]}$ A previous simulation study suggested that an eight-week gap between laser sessions may be more effective. ${ }^{[5]}$ The same trend was noticed in our simulations too. However, this effect may be related to the exclusion of catagen phase from the model.

Statistical models for prediction and optimization of LHR already exists. Monte Carlo simulation method is a popular and effective method for similar tasks. ${ }^{[6]}$ However, most of these simulations are research tools which cannot be used by a physician for patient education.

Agent-based modeling has been used for the study of complex systems in several fields including immunology. ${ }^{[7,20]}$ The intrinsic modular nature of $\mathrm{ABM}$ helps in representing a complex system like perturbations of hair growth cycle following the administration of an external agent like laser. Besides, the visual interface provided by the NetLogo ${ }^{[9]}$ makes the simulation easy to use and understand. However, the model uses random numbers and a particular simulation run is not exactly reproducible.

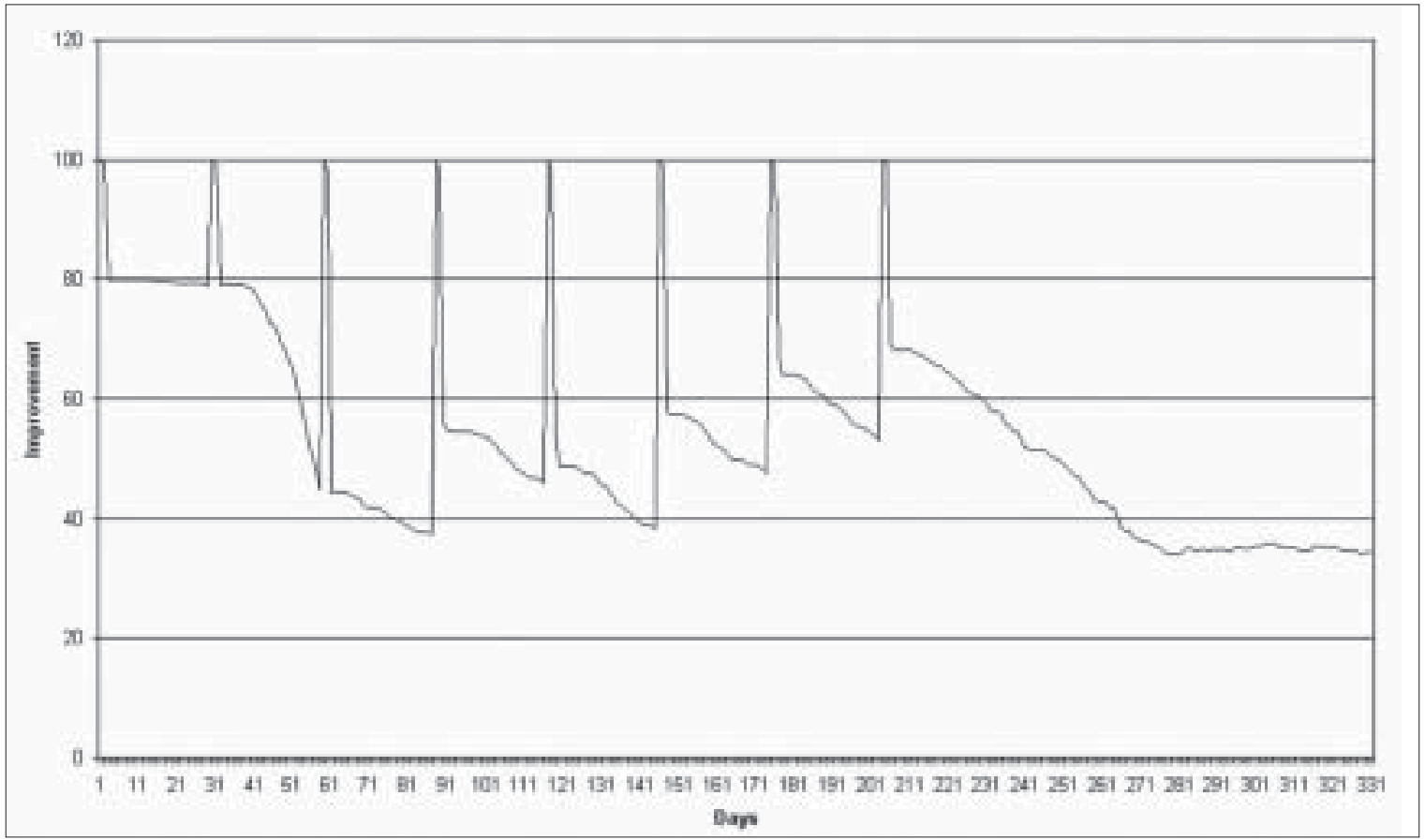

Figure 2: Plot of improvement vs. days during one of the simulations 
The model is based on several approximations. Catagen stage is not included in the model for simplification. Hairs in catagen stage are not affected by laser. Hence the exclusion of catagen, though small in number compared to other stages may affect the sensitivity of the model. The probability of injury for a telogen hair is known to be lower than anagen hair. ${ }^{[11]}$ However to simplify the model, we have used the same probability distribution. The only difference in the model between these two stages is the absence of anagen to telogen switching for a telogen hair. Pulse duration and spot size though included in the formula, is not modeled at present and is considered to be constant because of lack of reliable data. In our model, the hair thickness is ranked as thin and thick and the hair pigmentation as fine and dark to make calculations less complicated. However, representing these factors on a scale of 1 to 10 may be more accurate.

We also faced difficulty in assessing the overall improvement following laser. Clinical scales like the Ferriman-Gallway score ${ }^{[10]}$ is not sensitive enough for the purpose. Many of the existing simulations evaluate improvement based on the decrease in hair density. We believe that clinical improvement depends on several factors including hair density, length of visible hair, thickness and pigmentation of the hair. Hence we formulated an index based on our assessment of the importance of each of these factors and used that as a measure of improvement.

This model can be further extended by including less frequently encountered phenomena like paradoxical stimulation and phase synchronization. ${ }^{[21]}$ Catagen stage can be included in the model along with pulse duration and spot size.

\section{CONCLUSION}

Since undertaking clinical studies of LHR is a daunting task, most of the related beliefs are based on anecdotal reports or personal experience. Mathematical models, with all its limitations, can at the very least ask few questions about the validity of these beliefs. Agent- based modeling simplifies modeling and simulation, making it useful for physicians and even patients.

\section{REFERENCES}

1. Buddhadev RM. Standard guidelines of care: Laser and IPL hair reduction. Indian J Dermatol Venereol Leprol 2008;74:S68-74.

2. Zins JE, Alghoul M, Gonzalez AM, Strumble P. Self-reported outcome after diode laser hair removal. Ann Plast Surg 2008;60:233-8.

3. Casey AS, Goldberg D. Guidelines for laser hair removal. J Cosmet Laser Ther 2008;10:24-33.

4. Levy JL, Trelles MA, de Ramecourt A. Epilation with a longpulse $1064 \mathrm{~nm}$ Nd:YAG laser in facial hirsutism. J Cosmet Laser Ther 2001;3:175-9.

5. Kolinko V, Littler CM. Mathematical modeling for the prediction and optimization of laser hair removal. Lasers Surg Med 2000;26:164-76.

6. Roersma ME, Veldhuis GJ. Proposal and evaluation of a Monte Carlo model for hair regrowth following plucking. Skin Res Technol 2001;7:176-83.

7. An G. Introduction of an agent-based multi-scale modular architecture for dynamic knowledge representation of acute inflammation. Theor Biol Med Model 2008;5:11.

8. Sklar E. NetLogo, a multi-agent simulation environment. Artif Life 2007;13:303-11.

9. Wilensky U. NetLogo. Available at: http://ccl.northwestern.edu/ netlogo/ Accessed 24/10/2008.

10. Wild RA, Vesely S, Beebe L, Whitsett T, Owen W. Ferriman Gallwey self-scoring I: Performance assessment in women with polycystic ovary syndrome. J Clin Endocrinol Metab 2005;90:4112-4

11. Kolinko VG, Littler CM, Cole A. Influence of the anagen: Telogen ratio on Q-switched Nd:YAG laser hair removal efficacy. Lasers Surg Med 2000;26:33-40.

12. Rao J, Goldman MP. Prospective, comparative evaluation of three laser systems used individually and in combination for axillary hair removal. Dermatol Surg 2005;31:1671-6.

13. Rogachefsky AS, Becker K, Weiss G, Goldberg DJ. Evaluation of a long-pulsed Nd:YAG laser at different parameters: An analysis of both fluence and pulse duration. Dermatol Surg 2002;28:932-5.

14. Nouri K, Chen H, Saghari S, Ricotti CA, Jr. Comparing 18- versus $12-\mathrm{mm}$ spot size in hair removal using a gentlease $755 \mathrm{~nm}$ alexandrite laser. Dermatol Surg 2004;30:494-7.

15. Bouzari N, Tabatabai H, Abbasi Z, Firooz A, Dowlati Y. Laser hair removal: Comparison of long-pulsed Nd:YAG, long-pulsed alexandrite, and long-pulsed diode lasers. Dermatol Surg 2004;30:498-502.

16. Watanabe S. Basics of laser application to dermatology. Arch Dermatol Res 2008;300 Suppl 1:S21-30.

17. Lu SY, Lee CC, Wu YY. Hair removal by long-pulse alexandrite laser in oriental patients. Ann Plast Surg 2001;47:404-11.

18. Alster TS, Bryan $\mathrm{H}$, Williams CM. Long-pulsed Nd:YAG laser-assisted hair removal in pigmented skin: A clinical and histological evaluation. Arch Dermatol 2001;137:885-9.

19. Nanni CA, Alster TS. Laser-assisted hair removal: Side effects of Q-switched Nd:YAG, long-pulsed ruby, and alexandrite lasers. J Am Acad Dermatol 1999;41:165-71.

20. An G. Concepts for developing a collaborative in silico model of the acute inflammatory response using agent-based modeling. J Crit Care 2006;21:105-10.

21. Lolis MS, Marmur ES. Paradoxical effects of hair removal systems: A review. J Cosmet Dermatol 2006;5:274-6. 